\title{
An Approach for Obtaining Unique Kinematic Solutions of a Spherical Parallel Manipulator
}

\author{
Aibek Niyetkaliyev and Almas Shintemirov, Member, IEEE
}

\begin{abstract}
In this paper an approach for obtaining unique solutions to forward and inverse kinematics of a spherical parallel manipulator (SPM) system with revolute joints is proposed. Kinematic analysis of a general SPM with revolute joints is revisited and the proposed approach is formulated in the form of easy-to-follow algorithms that are described in detail. A graphical verification method using SPM computer-aideddesign (CAD) models is presented together with numerical and experimental examples that confirm the correctness of the proposed approach. It is expected that this approach can be applied to SPMs with different geometries and can be useful in designing real-time control systems of SPMs.
\end{abstract}

\section{INTRODUCTION}

Parallel manipulators are widely used for high speed, high accuracy positioning with limited workspace applications, e.g. in flight and automobile simulators, medical and industrial robotics, mechatronic applications, etc [1]-[3]. Among numerous types of parallel manipulators, spherical parallel manipulators (SPMs) can be applied for designing orientation wrist platforms for industrial robot end effectors, solar or parabolic antenna orientation systems, medical and rehabiliation robots [4]-[7]. A special configuration of an SPM with revolute joints named the Agile Eye is proposed in [8] for designing a three degree-of-freedom (DOF) optimal camera orientation system. The modification of the Agile Eye, the Agile Wrist, with enhanced load-carrying capacity and reduced weight is extensively studied in [9]-[13].

Many approaches were applied to analyse different configurations of SPMs [4]-[16]. It is shown in [4] that direct kinematic problem of a general SPM with revolute joints leads to a polynomial with at most eight solutions corresponding to different poses of the manipulator top platform. Practically, this polynomial is very complex and cannot be used for manipulator control system design. Multiple solutions exist in the SPM inverse kinematic problem. Nevertheless, unique solutions to SPM forward and inverse kinematics are required for designing real-time control systems of manipulators.

Considering special SPM configurations, e.g. the Agile Wrist/Eye, the complexity of direct and inverse kinematic problems reduces significantly and a closed form solutions can be obtained [16]. An approach for producing a unique solution to forward kinematics of the Agile Eye manipulator is reported in [17]. The reported approach was applied only

\footnotetext{
*This work was supported by the Kazakhstan Ministry of Science and Education within grant and target funding scheme (agreement \#001-2014)

The authors are with the Department of Robotics and Mechatronics, and Nazarbayev University Research and Innovation System, Nazarbayev University, 53 Kabanbay batyr Ave, Astana, 010000 Kazakhstan aibek.niyetkaliyev@nu.edu.kz, ashintemirov@nu.edu.kz
}

to one particular geometry of SPMs, i.e. the Agile Eye. Moreover, the mentioned CAD based verification procedure and identification of a unique solution to inverse kinematics of the SPM are not considered in that work.

In this paper the kinematic analysis of 3-DOFs SPMs with revolute joints is revisited and an approach for obtaining unique solutions to forward and inverse kinematics of a general SPM with revolute joints is proposed and discussed in detail. A graphical verification method using CAD software is presented together with a number of numerical examples that confirm the correctness of the proposed approach.

\section{KINEMATIC ANALYSIS OF A SPHERICAL PARALLEL MANIPULATOR}

The SPM kinematic analysis was extensively conducted previously, e.g. in works [4]-[18]. Below, a brief summary of SPM kinematics is introduced for completeness of the developments and results presented in this paper.

\section{A. Coordinate System}

A model of a general symmetric SPM with revolute joints is shown in Fig. 1. The SPM consists of two pyramid-shape platforms, a base and a top mobile platforms, connected by three equally spaced legs, numbered by $i=1,2,3$, each having two curved links. The axes of all joints, denoted by unit vectors $\mathbf{u}_{i}, \mathbf{v}_{i}$, and $\mathbf{w}_{i}$, intersect at a common center point, which is called the center of rotation. The dimensions of the proximal and distal links are assumed to be $\alpha_{1}$ and $\alpha_{2}$, respectively. Angles $\beta$ and $\gamma$ define the geometry of two regular pyramids of the base and the top mobile platforms. The motion of the top mobile platform is confined on the surface of a sphere centered at the center of rotation.

The right-handed orthogonal coordinate system with its origin located at the SPM rotation center is shown in Fig. 1. Axis $z$ is normal to the base pyramid platform and is directed upwards, while axis $y$ is located in the plane made by $z$ and $\mathbf{u}_{1}$. Input joint angles $\theta_{i}, i=1,2,3$, are measured from the plane made by $z$ axis and $\mathbf{u}_{i}$ to the plane of a lower link.

Unit vectors $\mathbf{u}_{i}, i=1,2,3$,for the axes of base joints are defined as follows [10]:

$$
\mathbf{u}_{i}=\left[\begin{array}{lll}
\sin \eta_{i} \sin \gamma, & \cos \eta_{i} \sin \gamma, \quad-\cos \gamma
\end{array}\right]^{T},
$$

where $\eta_{i}, i=1,2,3$, are the angles between projections of axes of the actuated revolutes on the base plane of the fixed pyramid and a given reference in that plane. By symmetry [5]

$$
\eta_{i}=2(i-1) \pi / 3
$$




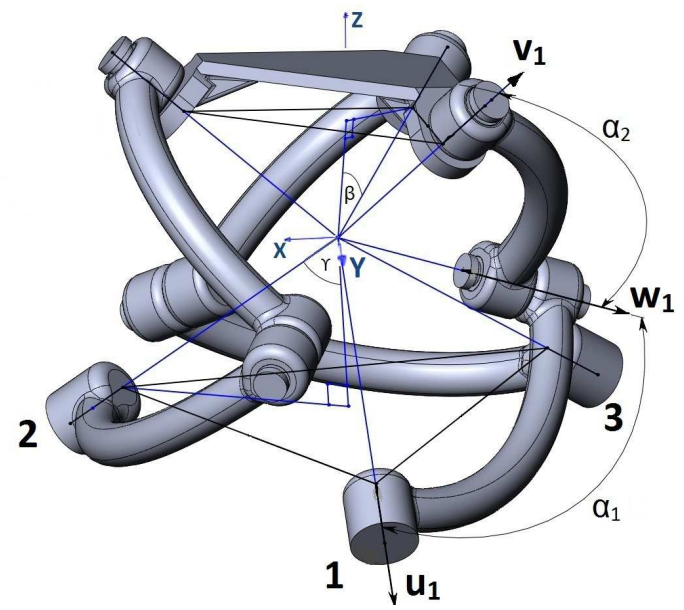

Fig. 1. Kinematic model of a spherical parallel manipulator.

Unit vectors $\mathbf{w}_{i}, i=1,2,3$, correspond to the axes of SPM intermediate revolute joints and are obtained in terms of input joint angles $\theta_{i}$ as below [10]:

$$
\mathbf{w}_{i}=\left[\begin{array}{c}
s \eta_{i} s \gamma c \alpha_{1}-\left(c \eta_{i} s \theta_{i}-s \eta_{i} c \gamma c \theta_{i}\right) s \alpha_{i} \\
c \eta_{i} s \gamma c \alpha_{1}+\left(s \eta_{i} s \theta_{i}+c \eta_{i} c \gamma c \theta_{i}\right) s \alpha_{i} \\
-c \gamma c \alpha_{1}+s \gamma c \theta_{i} s \alpha_{i}
\end{array}\right]
$$

where $s$ and $c$ denote sine and cosine, respectively.

Unit vectors $\mathbf{v}_{i}, i=1,2,3$, that are parallel to the axes of SPM top revolute joints, define orientation of the SPM top mobile platform.

\section{B. Forward Kinematics}

Considering the SPM forward kinematics problem, both $\mathbf{u}_{i}$ and $\mathbf{w}_{i}$ are assumed to be known. The task is to find unknown vectors $\mathbf{v}_{i}$ [18].

For the closed loop chain of SPM the following constraint equations hold [10]:

$$
\mathbf{w}_{i} \cdot \mathbf{v}_{i}=\cos \alpha_{2}, \quad i=1,2,3 .
$$

The geometry of the regular pyramid of the SPM top mobile platform implies [10]

$$
\mathbf{v}_{i} \cdot \mathbf{v}_{j}=\cos \alpha_{3}, \quad i, j=1,2,3, \quad i \neq j
$$

where $\alpha_{3}$ is the angle between axes of the $i$ th and $j$ th top joints. It is equal to the angle made by lateral edges of the top pyramid, which takes the value

$$
\alpha_{3}=2 \sin ^{-1}\left(\sin \beta \cos \frac{\pi}{6}\right) .
$$

Furthermore, unit vectors $\mathbf{v}_{i}$ lead to [10]

$$
\left\|\mathbf{v}_{i}\right\|=1 \text {. }
$$

There are several different approaches exist to obtain solutions to SPM forward kinematics problem. In this study, a combination of equations (4), (5) and (7) is utilized to generate a system of three linear and six quadratic equations of vectors $\mathbf{v}_{i}$ [10]. This system of non-linear equations with 9 unknowns (components of three $\mathbf{v}_{i}$ vectors) is solved using a numerical method, which allows obtaining a unique solution to the SPM forward kinematics problem as shown in the subsequent sections of this study.

Components of normal vector $\mathbf{N}$ to the SPM top mobile platform are defined as sums of the corresponding components of vectors $\mathbf{v}_{i}$. Thus, the normal unit vector $\mathbf{n}$ is found as

$$
\mathbf{n}=\frac{\mathbf{N}}{\|\mathbf{N}\|}
$$

where $\mathbf{N}=\left[v_{1 x}+v_{2 x}+v_{3 x}, v_{l y}+v_{2 y}+v_{3 y}, v_{1 z}+v_{2 z}+v_{3 z}\right]^{T}$.

Normal unit vector $\mathbf{n}=[0,0,1]^{T}$ if the SPM top mobile platform is parallel to the base platform in the coordinate system specified in Section II-A.

\section{Inverse Kinematics}

If the orientation of the SPM mobile platform is given, i.e., vectors $\mathbf{v}_{i}$ are known, inverse kinematic solutions are defined by three uncoupled equations for actuated joint angles $\theta_{i}$, as follows [13]:

$$
A_{i} T_{i}^{2}+2 B_{i} T_{i}+C_{i}=0, \quad i=1,2,3,
$$

with

$$
\begin{aligned}
T_{i}= & \tan \left(\frac{\theta_{i}}{2}\right) \\
A_{i}= & \left(-s \eta_{i} s \gamma c \alpha_{i}+s \eta_{i} c \gamma s \alpha_{i}\right) \cdot-v_{i x}+ \\
& +\left(c \eta_{i} s \gamma c \alpha_{i}-c \eta_{i} c \gamma s \alpha_{i}\right) \cdot v_{i y}+ \\
& +\left(c \gamma c \alpha_{i}-s \gamma s \alpha_{i}\right) \cdot v_{i z}-\cos \alpha_{2} ; \\
B_{i}= & c \eta_{i} s \alpha_{i} \cdot-v_{i x}+s \eta_{i} s \alpha_{i} \cdot v_{i y} ; \\
C_{i}= & \left(-s \eta_{i} s \gamma c \alpha_{i}-s \eta_{i} c \gamma s \alpha_{i}\right) \cdot-v_{i x}+ \\
& +\left(c \eta_{i} s \gamma c \alpha_{i}+c \eta_{i} c \gamma s \alpha_{i}\right) \cdot v_{i y}+ \\
& +\left(-c \gamma c \alpha_{i}+s \gamma s \alpha_{i}\right) \cdot v_{i z}-\cos \alpha_{2},
\end{aligned}
$$

where $v_{i x}, v_{i y}$, and $v_{i z}$ are the components of vector $\mathbf{v}_{i}$.

Equation (9) provides two solutions of angle $\theta_{i}$ for any given orientation of the SPM top mobile platform [4]. Hence, in total, there are eight solutions to inverse kinematics problem.

\section{PROPOSED APPROACH}

Using the kinematic model of a general SPM with revolute joints outlined in Section II, an approach for obtaining unique forward and inverse kinematic solutions of a SPM is proposed in this section. The approach is defined for a certain initial assembly of a SPM with all proximal links rotated to one side and attached to the base platform through actuated joints, e.g. as shown in Fig. 1. The approach is presented below in the form of the algorithms for obtaining unique forward and inverse kinematic solutions of a general SPM.

\section{A. Algorithm for Obtaining a Unique Forward Kinematics Solution for a SPM}

A unique forward kinematics solution in the form of vectors $\mathbf{v}_{i}$ is obtained as follows.

1) Use active joint angles $\theta_{i}$ :

- Calculate vectors $\mathbf{u}_{i}$ using equation (1) (optional).

- Calculate vectors $\mathbf{w}_{i}$ using equation (3). 
2) Construct a system of equations using equations (4), (5) and (7): Equation (4) results to three linear equations, whereas equations (5) and (7) give three quadratic equations each. Thus, in total a system of equations with 9 unknown components of three vectors $\mathbf{v}_{i}$ is derived.

3) Solve the system of equations using a numerical method to obtain vectors $\boldsymbol{v}_{i}$ : A lot of numerical methods require initial input values to solve a system of equations. In the present case, these values are the initial guesses of components $x, y$ and $z$ of unit vectors $\mathbf{v}_{i}, i=1,2,3$. Therefore, the signs of these components depend on the accepted coordinate system.

Use an initial guess vector with a certain sequence of signs to obtain a current unique solution for vectors $\mathbf{v}_{i}$. In the initial guess vector absolute values can be chosen arbitrary, since iterative numerical methods normally converge even if initial values are far from the solution.

On the other hand, signs of values in the initial guess vector are important. By changing the signs all possible eight solutions for the SPM forward kinematics, i.e. vectors $\mathbf{v}_{i}$, can be found. The correct sequence of signs corresponding to one current real solution can be selected using the orientation of vectors $\mathbf{v}_{i}$ of a SPM in its reference configuration specified in the accepted coordinate system. It can then be also verified with vectors $\mathbf{v}_{i}$ obtained from the SPM CAD model using the method presented in Section III-C.

The forward kinematics numerical example in Section IVA reveals the numerical method and the initial guess vector applied for calculations in this study.

\section{B. Algorithm for Obtaining a Unique Inverse Kinematics Solution for a SPM}

Assuming that the same initial SPM assembly and a coordinate system are considered for obtaining unique solutions to the manipulator forward and inverse kinematics, actuated joint angles $\theta_{i}, i=1,2,3$, can be found knowing vectors $\mathbf{v}_{i}$ corresponding to the current orientation of SPM top mobile platform as follows:

1) Calculate $A_{i}, B_{i}$ and $C_{i}$ using equation (11).

2) Solve equation (9) for $T_{i}$.

3) Find $\theta_{i}$ from equation (10) using positive roots of equation (9), i.e. positive values of $T_{i}$. The positive roots are considered due to accepted SPM initial configuration (working mode) where all three proximal links are rotated to a positive direction of actuated joints.

This algorithm can also be used for verification of unique forward kinematics solution obtained using the algorithm described in Section III-A.

\section{Graphical Identification of SPM Orientation with CAD Model}

The proposed approach is verified using a graphical method for identifying SPM orientation using CAD models. The method can be applied to various CAD software. In this study the SolidWorks software is used to briefly formulate the method as follows.

1) All three input joint angles of a SPM are specified between predefined planes and are fixed using the

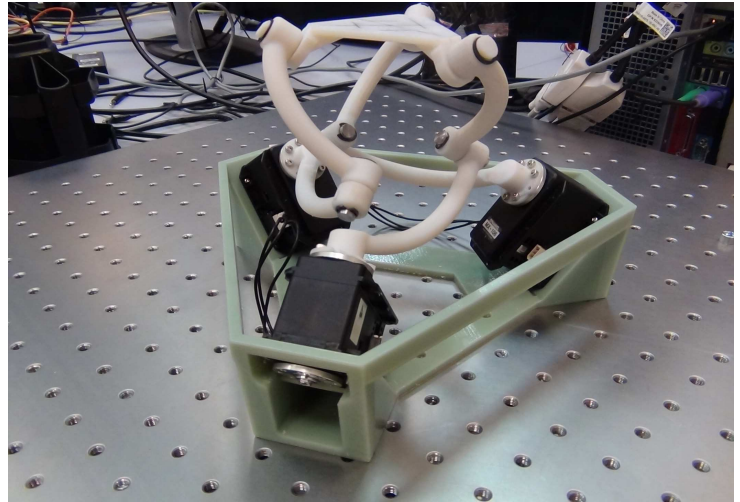

Fig. 2. A 3D printed prototype of the Agile Wrist manipulator.

Angle Mate tool available in the SolidWorks software. Thus, a fixed orientation of a SPM is obtained.

2) Using the 3D Sketch tool all axes of joints are connected with centerlines and the intersection point of these axes is defined as the center of rotation of a SPM.

3) The center of rotation is used as the origin point to attach the SPM coordinate system (defined in Section II-A) employing the Reference Geometry tool.

4) Three points are sketched on the top joints axes on the distance of one unit $(1 \mathrm{~mm})$ from the coordinate system origin.

5) Three unit vectors $\mathbf{v}_{i}, i=1,2,3$, are specified and their coordinates relative to the inserted coordinate system can be obtained using the Measure tool.

As a result, the SPM orientation, corresponding to three input joint angles $\theta_{i}$, is obtained in terms of three unit vectors $\mathbf{v}_{i}, i=1,2,3$.

\section{NUMERICAL EXAMPLES AND DISCUSSION}

To demonstrate application of the proposed approach, authors designed a SolidWorks CAD model and a 3D printed prototype of the Agile Wrist manipulator. The prototype includes three Dynamixel MX-106 servomotors fixed to the base platform of the SPM and is assembled as shown in Fig. 2. In the Agile Wrist SPM configuration $\alpha_{1}=\alpha_{2}=90 \mathrm{deg}$. with all three legs being identical. Three unit vectors $\mathbf{u}_{i}$ as well as vectors $\mathbf{v}_{i}, i=1,2,3$, are mutually orthogonal [10]. This is resulted to $\beta=\gamma=54.75 \mathrm{deg}$.

The reference configuration of the Agile Wrist is chosen such that all active joint angles $\theta_{1}=\theta_{2}=\theta_{3}=135 \mathrm{deg}$. and the axes of base and top joints are aligned as follows:

$$
\mathbf{u}_{1}=-\mathbf{v}_{3}, \quad \mathbf{u}_{2}=-\mathbf{v}_{1}, \quad \mathbf{u}_{3}=-\mathbf{v}_{2} .
$$

In this reference configuration, the SPM top mobile and base platforms are parallel to each other as illustrated in Fig. 2.

\section{A. Calculating Orientation of the Agile Wrist}

Consider the case with input angles $\theta_{1}=95 \mathrm{deg}$., $\theta_{2}=110 \mathrm{deg}$. and $\theta_{3}=105 \mathrm{deg}$. which corresponds to the top platform orientation shown in Fig. 3. 


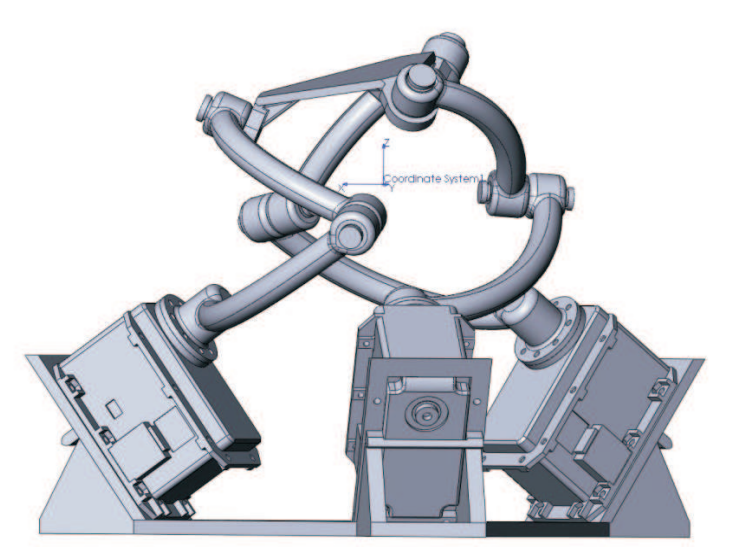

Fig. 3. Orientation of the Agile Wrist SPM corresponding to actuated joint angles $\theta_{1}=95$ deg., $\theta_{2}=110 \mathrm{deg}$. and $\theta_{3}=105 \mathrm{deg}$.

Following the algorithm outlined in Section III-A all unit vectors $\mathbf{u}_{i}$ and $\mathbf{w}_{i}$ are calculated in MATLAB using equations (1) and (3) as below:

$$
\mathbf{u}_{1}=\left[\begin{array}{c}
0 \\
0.8166 \\
-0.5771
\end{array}\right], \mathbf{u}_{2}=\left[\begin{array}{c}
0.7072 \\
-0.4083 \\
-0.5771
\end{array}\right], \mathbf{u}_{3}=\left[\begin{array}{c}
-0.7072 \\
-0.4083 \\
-0.5771
\end{array}\right]
$$

and

$$
\mathbf{w}_{1}=\left[\begin{array}{l}
-0.9962 \\
-0.0503 \\
-0.0712
\end{array}\right], \mathbf{w}_{2}=\left[\begin{array}{c}
0.2989 \\
0.9125 \\
-0.2793
\end{array}\right], \mathbf{w}_{3}=\left[\begin{array}{c}
0.6123 \\
-0.7618 \\
-0.2114
\end{array}\right] .
$$

Then, combining equations (4), (5) and (7) the system of three linear and six quadratic equations is generated as follows:

$$
\left\{\begin{array}{l}
-0.9962 v_{1 x}-0.0503 v_{1 y}-0.0712 v_{1 z}=0 \\
0.2989 v_{2 x}+0.9125 v_{2 y}-0.2793 v_{2 z}=0 \\
0.6123 v_{3 x}-0.7618 v_{3 y}-0.2114 v_{3 z}=0 \\
v_{1 x} \cdot v_{2 x}+v_{1 y} \cdot v_{2 y}+v_{1 z} \cdot v_{2 z}=0 \\
v_{1 x} \cdot v_{3 x}+v_{1 y} \cdot v_{3 y}+v_{1 z} \cdot v_{3 z}=0 \\
v_{2 x} \cdot v_{3 x}+v_{2 y} \cdot v_{3 y}+v_{2 z} \cdot v_{3 z}=0 \\
v_{1 x}^{2}+v_{1 y}^{2}+v_{1 z}^{2}=1 \\
v_{2 x}^{2}+v_{2 y}^{2}+v_{2 z}^{2}=1 \\
v_{3 x}^{2}+v_{3 y}^{2}+v_{3 z}^{2}=1
\end{array}\right.
$$

The system of non-linear equations (12) with 9 unknown components of vectors $\mathbf{v}_{i}$ is solved in MATLAB using function fsolve with the initial guess vector

$$
x_{0}=[-1,1,1,1, \pm 1,1,-1,-1,1]^{T} .
$$

corresponding to vector $\left[v_{1 x}, v_{1 y}, v_{1 z}, v_{2 x}, v_{2 y}, v_{2 z}, v_{3 x}, v_{3 y}, v_{3 z}\right]^{T}$.

Function fsolve uses the trust-region-dogleg algorithm [19] that requires initial input values to solve a system of equations. The absolute values in vector $x_{0}$ were chosen arbitrarily, whereas the sequence of signs in vector $x_{0}$ was adopted from the orientation of vectors $\mathbf{v}_{i}, i=1,2,3$, at the Agile Wrist reference configuration and then verified with numerous tests using the SolidWorks CAD software.

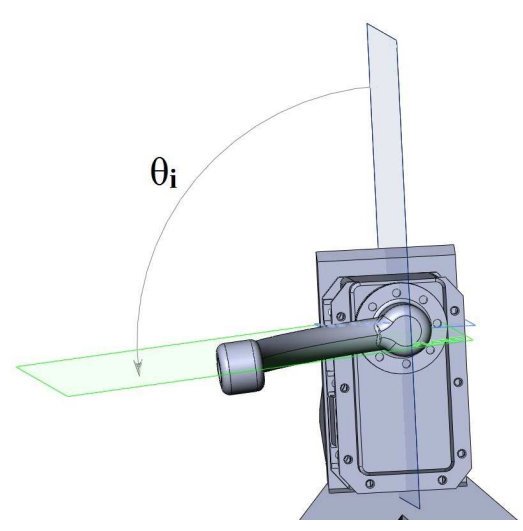

Fig. 4. Angle $\theta_{i}$ of a base actuated joint measured from the plane made by axis $z$ and vector $\mathbf{u}_{i}$ to the plane of a proximal link.

As a result, the orientation of the Agile Wrist top mobile platform corresponding to the given actuated joint angles is found in terms of unit vectors $\mathbf{v}_{i}$ :

$$
\mathbf{v}_{1}=\left[\begin{array}{c}
-0.0817 \\
0.8230 \\
0.5621
\end{array}\right], \mathbf{v}_{2}=\left[\begin{array}{c}
0.9039 \\
-0.1768 \\
0.3896
\end{array}\right], \mathbf{v}_{3}=\left[\begin{array}{c}
-0.4204 \\
-0.5401 \\
0.7291
\end{array}\right]
$$

Verification of the unique forward kinematics solution (14) of the considered SPM can be done using the graphical method described in Section III-C. The procedure using the SolidWorks is described below.

At first, the input joint angles are fixed using the Angle Mate feature. The angle $\theta_{1}=95 \mathrm{deg}$. is set from the plane made by axis $z$ and vector $\mathbf{u}_{1}$ to the plane of a corresponding lower link in the direction shown in Fig. 4. Similarly, all three actuated joint angles are set in the Agile Wrist CAD model, ensuring the corresponding orientation of the top mobile platform as shown in Fig. 3.

Then, using the 3D Sketch tool an intersection point of axes of all SPM revolute joints is positioned. This point defines the center of rotation of the SPM. The Reference Geometry tool is used to attach the specified in Section II-A right-handed orthogonal coordinate system to the center of rotation.

Fig. 5 illustrates the centerlines of axes drawn from the origin of the coordinate system through centers of joints of the Agile Wrist top mobile platform. To specify unit vectors $\mathbf{v}_{i}$ three points are sketched on these centerlines of axes on 1 unit $(1 \mathrm{~mm})$ distance from the origin. XYZ coordinates of these vectors relative to the coordinate system origin are found using the Measure tool as shown in Fig. 6. In a similar manner all three unit vectors $\mathbf{v}_{i}$ are found as below:

$$
\mathbf{v}_{1}=\left[\begin{array}{c}
-0.0818 \\
0.8229 \\
0.5623
\end{array}\right], \mathbf{v}_{2}=\left[\begin{array}{c}
0.9037 \\
-0.1769 \\
0.3898
\end{array}\right], \mathbf{v}_{3}=\left[\begin{array}{c}
-0.4204 \\
-0.5402 \\
0.7292
\end{array}\right]_{(15)}
$$

It can be observed that the values of vectors $\mathbf{v}_{i}$ calculated in MATLAB, equation (14), match the results (15) obtained using the CAD model. Hence, the sequence of signs in the 


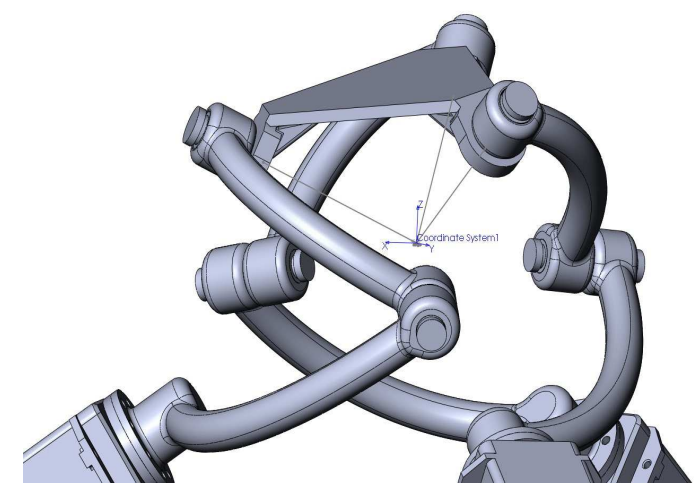

Fig. 5. Axes centerlines drawn in the SolidWorks using the 3D Sketch tool.

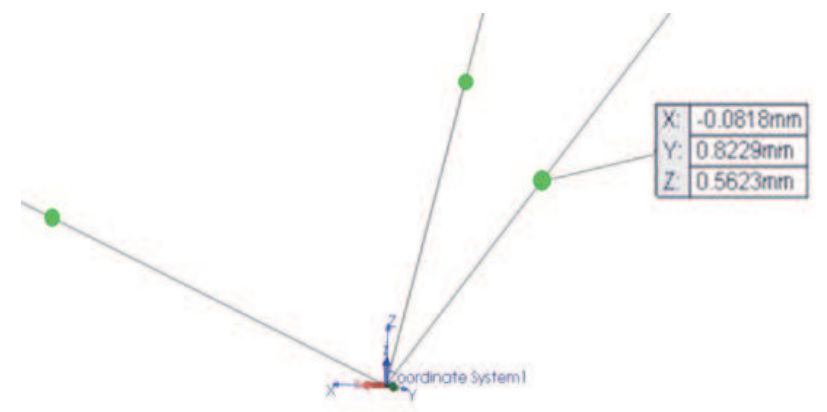

Fig. 6. Coordinates of vector $\mathbf{v}_{1}$.

initial guess vector $x_{0}$, defined in (13), ensures the correct unique forward kinematic solution and can be used for this SPM hereinafter in calculations.

In addition, normal unit vector $\mathbf{n}$ of the SPM top platform is defined from equation (8) as follows:

$$
\mathbf{n}=[0.2321,0.0613,0.9708]^{T} .
$$

Indeed, this normal unit vector $\mathbf{n}$ can also be defined graphically in the CAD model similarly to the way unit vectors $\mathbf{v}_{i}$ in (15) are obtained.

\section{B. Inverse Kinematic Solution of the Agile Wrist}

The unique Agile Wrist inverse kinematic solution for the same SPM orientation can be obtained as described in Section III-B. Assuming that vectors $\mathbf{v}_{i}$ are given in the form of (14), coefficients (11) are calculated in MATLAB. Thus, three uncoupled quadratic equations (9) are written as follows:

$$
\begin{aligned}
& -0.9340 t_{1}^{2}+2 \cdot 0.0817 t_{1}+0.9340=0 \\
& -0.8209 t_{2}^{2}+2 \cdot 0.2988 t_{2}+0.8209=0 \\
& -0.9614 t_{3}^{2}+2 \cdot 0.2576 t_{3}+0.9614=0 .
\end{aligned}
$$

From equation (16) the inverse kinematics solutions are calculated using equation (10) in terms of actuated joint angles $\theta_{i}$ in degrees as follows:

$\theta_{1}=\left[\begin{array}{c}-85.0002 \\ 94.9998\end{array}\right], \theta_{2}=\left[\begin{array}{c}-70.0002 \\ 109.9998\end{array}\right], \theta_{3}=\left[\begin{array}{c}-75.0002 \\ 104.9998\end{array}\right]$.
TABLE I

THE RESULTS OF THE PROPOSED APPROACH AND THE APPROACH PRESENTED IN [17]

\begin{tabular}{l|c|c|c|c|c}
\hline \hline$\theta_{i \text { input }}$ & $\mathbf{v}_{1}$ & $\mathbf{v}_{2}$ & $\mathbf{v}_{3}$ & $\theta_{i n v}$ & $\mathbf{n}$ \\
\hline \hline \multicolumn{7}{c}{ Proposed Approach } \\
\hline 125 & -0.3643 & -0.0225 & -0.9308 & 125 & -0.7611 \\
90 & 0.9310 & 0.0130 & -0.3651 & 90 & 0.3344 \\
75 & -0.0207 & 0.9997 & -0.0166 & 75 & 0.5558 \\
\hline \hline \multicolumn{7}{c}{ Approach [17] } \\
\hline 10 & 0.7719 & -0.5664 & -0.2886 & - & - \\
45 & -0.6261 & -0.5986 & -0.4998 & - & - \\
60 & -0.1104 & -0.5664 & 0.8167 & - & - \\
\hline \hline
\end{tabular}

Analysing above calculated roots of equation (16), it can be observed that positive roots, i.e. positive actuated joint angles $\theta_{i}$, are equal to the initially given input angles $\theta_{1}=95$ deg., $\theta_{2}=110 \mathrm{deg}$. and $\theta_{3}=105 \mathrm{deg}$. in this example. Hence, it can be concluded that the above obtained unique forward kinematic solution, vectors $\mathbf{v}_{i}$ in (14), is correct. Note that there is $180 \mathrm{deg}$. difference between negative and positive joint angles $\theta_{i}$.

Similar numerical and graphical analyses for a number of different orientations of the Agile Wrist SPM confirmed the validity of the proposed approach. Calculated kinematic solutions are used for motion generation of the Agile Wrist prototype which can be viewed in the accompanying video available at www.alaris.kz.

\section{Comparison with the Approach [17]}

The proposed approach was compared with the approach for obtaining a unique solution to forward kinematics of the Agile Eye presented in [17]. To facilitate the analysis in that work, two orthogonal coordinate systems are set up along the axes of top and base platform joints of the Agile Eye, respectively. At the reference orientation these coordinate systems coincide with each other and input joint angles are all equal to 0 . This reference orientation of the Agile Eye is equivalent to the reference orientation of the Agile Wrist CAD model, adopted in this paper, with all input joint angles equal to $135 \mathrm{deg}$. as defined in Section IV-A. For comparison purposes the transformation matrix $\mathrm{T}$ from the coordinate system defined in Section II-A to the one defined in [17] is derived in degrees as follows:

$$
\mathbf{T}=\left[\begin{array}{ccc}
\cos 90 & \cos 35.3 & \cos 125.3 \\
\cos 45 & \cos 114.1 & \cos 125.3 \\
\cos 135 & \cos 114.1 & \cos 125.3
\end{array}\right]
$$

Authors considered a number of different sets of input actuated joint angles $\theta_{i \text { input }}, i=1,2,3$. The calculated results using both the approaches for one case are presented in Table I. Fig. 7 illustrates the SPM configuration corresponding to this case. The upper part of Table I contains the results obtained using the approach proposed in this work. The lower part of the table shows the values calculated according to the formula presented in [17]. The input joint 


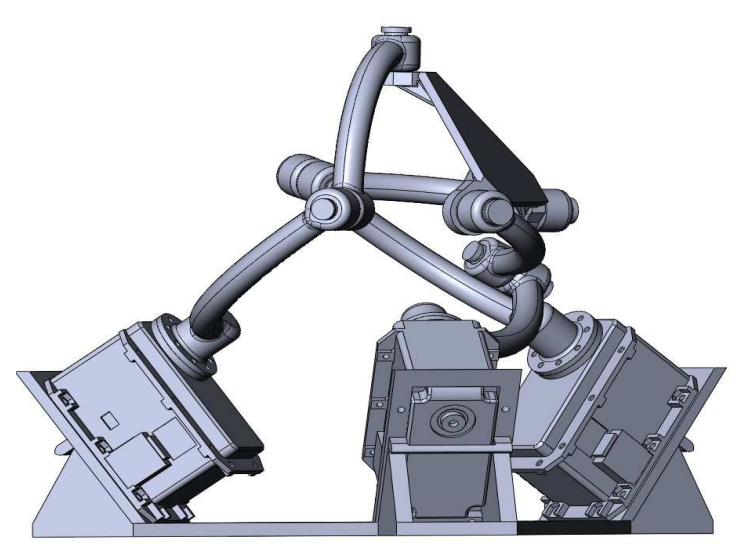

Fig. 7. The SPM configuration corresponding to Table I.

angles corresponding to the same SPM pose are given in degrees and coordinates of unit vectors $\mathbf{v}_{i}$ are provided in two different coordinate systems corresponding to the two approaches, respectively. Transformation matrix (17) can be used to mathematically verify that vectors $\mathbf{v}_{i}$ obtained by both the approaches represent the same orientation of the SPM. The results are also verified with the graphical method described in Section III-C by attaching additional coordinate system defined in [17].

Analysis of the calculated results in Table I shows that the computations using both the approaches result in a correct unique forward kinematic solution of the considered SPM in terms of unit vectors $\mathbf{v}_{i}$. In addition, the proposed approach allows calculating a unique inverse kinematic solution and a normal unit vector of the SPM top mobile platform.

\section{CONCLUSIONS AND FUTURE WORK}

The proposed in this paper approach solves a challenging kinematic problem of identifying unique forward and inverse solutions of SPMs with revolute joints. A current forward kinematic solution can be derived from a system of nonlinear equations solved in MATLAB using a numerical iterative method with an initial guess vector. The sequence of signs in the initial guess vector is defined taking into account reference orientation of the SPM top mobile platform.

On the other hand, a unique inverse kinematic solution corresponds to positive roots of the SPM inverse kinematic equation. A graphical method for identifying SPM orientation using CAD models is presented and applied for verification of the obtained kinematic solutions. Numerical examples and comparison analysis with the Agile Wrist SPM demonstrate application and correctness of the proposed approach. Calculated unique kinematic solutions are implemented for motion trajectory generation of a 3D printed prototype of the Agile Wrist. The accompanying video of the prototype motion is available at www.alaris.kz.

It is expected that this approach can be applied to SPMs with different geometries and can be useful in designing manipulator real-time control systems. Further work include singularity and dynamic analysis of general SPM.

\section{REFERENCES}

[1] J. Pradipta, M. Klunder, M. Weickgenannt, and O. Sawodny, "Development of a pneumatically driven flight simulator Stewart platform using motion and force control," in IEEE/ASME International Conference on Advanced Intelligent Mechatronics (AIM), San Diego, California USA, 2013, pp. $158-163$.

[2] J. A. Saglia, N. G. Tsagarakis, J. S. Dai, and D. G. Caldwell, "Control strategies for patient-assisted training using the ankle rehabilitation robot (ARBOT)," IEEE/ASME Transactions on Mechatronics, vol. 18, no. 6, pp. 1799-1808, 2013.

[3] F. Pierrot, V. Nabat, S. Krut, and P. Poignet, "Optimal design of a 4-DOF parallel manipulator: From academia to industry," IEEE Transactions on Robotics, vol. 25, no. 2, pp. 213-224, 2009.

[4] C. Gosselin, J.Sefrioui, and M. Richard, "On the direct kinematics of spherical three-degree-of-freedom parallel manipulators of general architecture," ASME Journal of Mechanical Design, vol. 116, pp. 594598, 1994.

[5] C. Gosselin and E. Lavoie, "On the kinematic design of spherical three-degree-of-freedom parallel manipulators," The International Journal of Robotics Research, vol. 12, pp. 394-402, 1993.

[6] T. A. Hess-Coelho, "A redundant parallel spherical mechanism for robotic wrist applications," ASME Journal of Mechanical Design, vol. 129, pp. 891-895, 2007.

[7] M. Malosio, S. P. Negri, N. Pedrocchi, F. Vicentini, M. Caimmi, and L. M. Tosatti, "A spherical parallel three degrees-of-freedom robot for ankle-foot neuro-rehabilitation," in 34th Annual International Conference of the IEEE EMBS, San Diego, California USA, July 2012, pp. 3356-3359.

[8] C. Gosselin, E. St-Pierre, and M. Gagni, "On the development of the Agile Eye," IEEE Robotics \& Automation Magazine, vol. 3, pp. 29-37, 1996.

[9] F. Bidault, C.-P. Teng, and J. Angeles, "Structural optimization of a spherical parallel manipulator using a two-level approach," in Proceedings of ASME Design Engineering Technical Conferences and Computers and Information in Engineering Conference, Pittsburgh, Pennsylvania, 2001.

[10] S. Bai, M. R. Hansen, and J. Angeles, "A robust forward-displacement analysis of spherical parallel robots," Mechanism and Machine Theory, vol. 44, pp. 2204-2216, 2009.

[11] K. Al-Widyan, X. Q. Mab, and J. Angeles, "The robust design of parallel spherical robots," Mechanism and Machine Theory, vol. 46, pp. 335-343, 2011.

[12] S. Staicu, "Recursive modelling in dynamics of Agile Wrist spherical parallel robot," Robotics and Computer-Integrated Manufacturing, vol. 25, pp. 409-416, 2009.

[13] Y. Jian and J. Angeles, "Kinematics simulation and control design of the Agile Wrist in a dual-arm robotic mechanical systems," in 34th Annual International Conference of the IEEE EMBS.

[14] S. Bai, M. R. Hansen, and T. O. Andersoen, "Modelling of a special class of spherical parallel manipulators with Euler parameters," Robotica, vol. 27, pp. 161-170, 2008.

[15] I. A. Bonev, D. Chablat, and P. Wenger, "Working and assembly modes of the Agile Eye," in Proceedings of the 2006 IEEE International Conference on Robotics and Automation, Orlando, Florida, May 2006, pp. 2317-2322.

[16] C. Gosselin and M. Gagne, "A closed-form solution for the direct kinematics of a special class of spherical three-degree-of-freedom parallel manipulators," in Proceedings of the Second Workshop on Computational Kinematics, Sophia Antipolis, France, September 1995, pp. 231-240.

[17] X. Kong and C. M. Gosselin, "A formula that produces a unique solution to the forward displacement analysis of a quadratic spherical parallel manipulator: The Agile Eye," Journal of Mechanisms and Robotics, vol. 2, pp. 044 501-1-044 501-4, 2010.

[18] S. Bai and M. R. Hansen, "Forward kinematics of spherical parallel manipulators with revolute joints," in Proceedings of the 2008 IEEE/ASME International Conference on Advanced Intelligent Mechatronics, Xi'an, China, July 2008, pp. 532-527.

[19] M. Powell, "A Fortran subroutine for solving systems of nonlinear algebraic equations," in Numerical Methods for Nonlinear Algebraic Equations, P. Rabinowitz, Ed., 1970, ch. 7. 